\title{
ABSORPTION-LINE REDSHIFTS OF GALAXIES IN REMOTE CLUSTERS OBTAINED WITH A SKY-SUBTRACTION SPECTROGRAPH USING AN SIT TELEVISION DETECTOR
}

\author{
J. A. Westphal, Jerome Kristian, and Allan Sandage \\ Hale Observatories, Carnegie Institution of Washington, California Institute of Technology \\ Received 1975 January 24
}

\begin{abstract}
A prism spectrograph with an associated SIT television camera, operating as a two-dimensional detector with digital readout, has been used at the 5-m Hale telescope. The system was tested for its ability to subtract the sky spectrum, and was found to produce difference spectra that are essentially photon noise limited.

Redshifts of 14 galaxies in clusters with $0.01 \leq z \leq 0.4$ were obtained, each with exposure times of 90 minutes or less. Nine of the redshifts are new. Redshifts for the remaining five agree with previous values to within the measuring errors.

The speed and sky-subtraction capabilities of the instrument are sufficient to begin routine measurement of absorption-line redshifts for remote cluster galaxies in an effort to extend the Hubble diagram.

Subject headings: galaxies, clusters of - redshifts
\end{abstract}

\section{INTRODUCTION}

Spectra for very faint objects cannot be obtained on photographic plates because the nightsky airglow saturates the emulsion before an acceptable signal-to-noise level is reached. For example, Humason (1957) was stopped in his program to measure redshifts of cluster galaxies at $z \equiv \Delta \lambda / \lambda_{0} \approx 0.2$. Plate III-8 of Humason, Mayall, and Sandage (1956) shows the severity of the problem, near sunspot maximum, for the Hydra cluster. The widespread use of image tubes during the 1960s, thanks in large part to the very successful efforts of the Carnegie Committee on Image Tubes for Telescopes (Carnegie Institution of Washington Yearbooks, 19531969), greatly increased the speed of obtaining spectra, but did not change the faintness limit, because the image tubes also used photographic methods to record the spectra.

In more recent years, the replacement of photographic emulsions by linear detectors with large dynamic range has made it possible in principle to obtain spectra for arbitrarily faint sources, limited in practice only by the time one is willing to devote to the observations, which is determined by the telescope aperture, the quantum efficiency of the detector, and the ultimate limit of the noise due to the random arrival of incoming photons. With a linear detector, the sky background can be subtracted, and exposures added together to increase signalto-noise ad lib. A wide variety of detectors have been built or proposed, including the Robinson-Wampler (1972) image-dissector scanner and a similar device developed independently by W. K. Ford, the BoksenbergBurgess (1973, quoted in Glaspey and Walker 1973) device, the Princeton SEC system (Lowrance and Zucchino 1969; Zucchino and Lowrance 1971), the Digicon (Beaver and McIlwain 1971), and others (cf. the proceedings of a conference on television-type sensors edited by Glaspey and Walker 1973).

The present paper gives the first results from a new system which uses an SIT (Silicon Intensifier Target) television camera tube as a detector.

\section{THE DIGITAL PRISM SPECTROGRAPH WITH SIT DETECTOR}

To measure redshifts of faint galaxies, we have built a system that consists of a prism spectrograph and an SIT television detector with digital output. A prism system was chosen to maximize the throughput for faint objects. The spectrograph design parameters were matched to the detector and optimized for the galaxy redshift problem. The projected slit width is about $60 \mu$ for a $1^{\prime \prime}$ slit opening, while the resolution element of the detector is $40 \mu$. One, two, or three prisms can be used to provide a range of dispersions. The two-prism configuration used for the measurements reported here gives a spectral resolution (including the detector) of $7 \AA$, with a dispersion of $125 \AA \mathrm{mm}^{-1}$, at $\mathrm{H} \gamma$.

The detector system was developed and is being used as an area photometer. A detailed description is given elsewhere (Westphal 1973, quoted in Glaspey and Walker 1973; Kristian and Westphal 1975). It uses a commercial 16-mm SIT television camera tube (RCA 4804 ) operated in an integrating mode. The front of the tube is a fiber-optic plate, with a S20 photocathode deposited on its inner surface. Photoelectrons liberated at the photocathode are accelerated through a $10-\mathrm{kV}$ field and electrostatically focused on a silicon wafer target. It is this target which gives the tube its unique properties: the electron acceleration section is similar to other image intensifiers.

The target contains about a million individual silicon diodes, on a rectangular grid with $10-\mu$ spacing. The target is back biased, and the diodes act as individual storage capacitors. The target is fully charged $\left(\sim 10^{5}\right.$ electrons per diode) before an exposure. Each accelerated photoelectron creates about 1000 electron-hole carrier pairs when it strikes the target. The holes then migrate to the diodes and partially discharge them. 


\section{WESTPHAL, KRISTIAN, AND SANDAGE}

Affter an exposure is made, the tube is read out by rechrarrging the target in a raster pattern. The charging curent at any place on the target is proportional to thes number of photons incident at the corresponding plate on the input face plate. The read beam is defocused so that the landing spot is about $40 \mu$ in diameter, which sets the basic resolution of the detector. The read beam current is amplified and A/D converted. The resulting 12-bit binary numbers, which are a linear representation of the two-dimensional intensity distribution of the spectrograph output, are recorded on digital magnetic tape for later reduction.

The readout noise is about 500 carriers rms per pixel ("pixel" is an acronym for picture element: i.e., resolution element). Because each photoelectron produces about 1000 carriers, it follows that individual photoelectrons can be detectable, and the system should be nearly photon-noise limited. This expectation is observed to be the case. The target saturates at a level of 1000 photoelectrons per pixel ( $10^{6}$ carriers), so that the dynamic range is about $10^{3}$. The output is very nearly proportional to the input, except for small deviations which are well-behaved and easily calibrated (Kristian and Westphal 1975).

It should be noted that the output is fully twodimensional, which has two major advantages for spectroscopy over a one-dimensional format: (1) The relative sensitivity of the tube can be calibrated transverse to the dispersion, so that the calibrated output does not depend upon the details of the light distribution along the slit, which will vary in an uncontrolled way due to seeing and tracking changes. (2) If a long slit is used, a large sample of sky can be measured simultaneously with the star or galaxy spectrum with high accuracy, by adding the large number of available sky pixels. This means that no extra telescope time is spent on sky measurements, and that sky subtraction can be done even with rapidly changing sky conditions.

\section{FIRST OBSERVATIONS}

The spectrograph and detector were first used at the Cassegrain focus of the Hale reflector in 1974 October and December. The aim of the observations was to test (1) the speed of the system, (2) the sky-subtraction capability, and (3) the suitability of the system for measuring absorption-line redshifts of faint galaxies. The October observations were marred by continuous variable cirrus cloud cover, heavy enough to prevent observations most of the time. These made the results of the speed tests only impressionistic, but provided an especially severe test for sky-subtraction. The December observations were made through thin clouds, with good seeing. Spectra and redshifts were obtained for galaxies in 14 clusters, with redshifts ranging from 0.013 to 0.392 .

Sky subtraction was done for each frame, using the data for that frame only. A long slit was used, and the sky spectrum determined by averaging over the parts of the slit away from the galaxy. The mean sky spectrum was then subtracted from the galaxy spectrum, point-by-point. Figure 1 shows the results of the skysubtraction for the cluster $0024+1654$.
The subtraction appears to work well on all the available data. In all cases, the bright emission lines from the airglow and from the city lights of San Diego were reduced to the photon noise level. In several cases, lines in the galaxy spectra were recovered at the same wavelength as a subtracted nightsky line. One example is the G-band in NGC 7619 (fig. 2), which was recovered from directly under the very bright $\lambda 4358$ mercury line. Sky-subtracted spectra for two additional galaxies are shown in figure 2 .

The three clusters shown in the diagrams are representative of the other data we obtained. They cover the range of redshifts $(z=0.01$ to 0.4$)$ measured here in these tests, and are also of historical interest. NGC 7619 was the prime test object used by Humason (1929) to prove his techniques before embarking on the longterm program of redshift measurements to extend Hubble's (1929) original velocity-distance relation. The redshift was twice as large as any obtained previously, and was measured from two spectra taken with the Mount Wilson 100-inch $(2.5 \mathrm{~m})$, with exposure times of 33 and 45 hours. For comparison, the spectrum shown in figure 2 was obtained in 30 minutes through several magnitudes of cloud cover. Abell 520, at $z=0.2$, is near the limit for absorption-line redshifts using photographic techniques. The cluster $0024+1654(V \approx 20 \mathrm{mag})$ was beyond Humason's reach with the 200 -inch $(5 \mathrm{~m})$ primefocus spectrograph (Humason 1957). It was one of the first objects measured by Baum (1962), using the technique of measuring redshifts from the shift of the galaxy continuum, and by Oke (1971), using the same technique with the 200-inch multichannel scanner.

The error bars in figures 1 and 2 show the expected rms fluctuations due to photoelectron counting statistics. These are comparable with the observed noise in the spectrum itself, indicating that the system is limited primarily by photon statistics rather than by internal instrumental noise.

The first redshifts measured with the SIT system are given in table 1 . The procedure for calibrating the wavelength scale is analogous to that used with photographic plates. Comparison spectra were recorded with each galaxy spectrum and used to determine wavelength as a function of position on the tube. Approximate redshifts $(\Delta z \sim 0.01)$ were first determined by direct visual inspection of the absorption-line wavelengths. More accurate values were then determined by cross-correlating the spectra against a high-resolution scan of the center of M31 (kindly supplied by Hyron Spinrad), numerically redshifted over an appropriate range, and degraded to our present spectral resolution. The final accuracy of the redshifts appears to be $z \leq 0.001$ in all cases. Of the 14 redshifts given in table 1 , five had been measured earlier by others using conventional techniques. Our results agree with the earlier values to within the combined errors.

The speed of the spectrograph appears to be satisfactory. Spectra with more than adequate signal-tonoise were obtained in 30 minutes for galaxies at $z=$ 0.2 and in 90 minutes for galaxies at $z=0.4$. Although it is difficult to make a quantitative statement without a direct measurement of the throughput, this seems to 


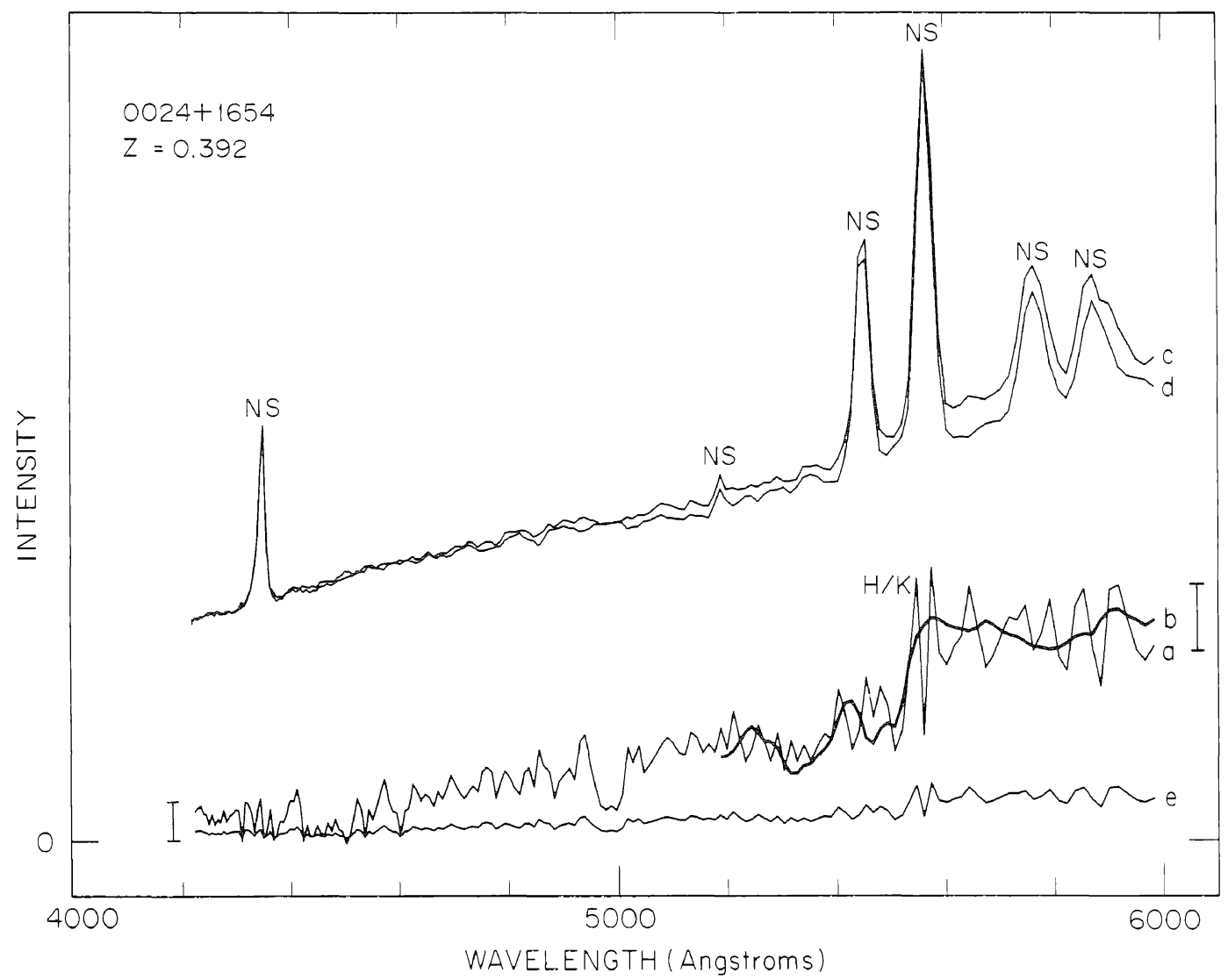

FIG. 1.--Digital prism spectrograph data for the cluster $0024+1654$, showing the sky subtraction capability of the instrument. Curve $c$ is the raw data for the galaxy spectrum, $90 \%$ or more of which is due to contamination from the night sky. The emission lines labeled "NS" are nightsky airglow and mercury lines from San Diego. Curve $d$ is the spectrum of the night sky alone, averaged over those parts of the spectrograph entrance slit away from the galaxy. Curve $e$ is the difference, $c-d$ which represents the uncontaminated galaxy spectrum. Curves $c, d$, and $e$ are on the same vertical intensity scale, with zero marked, while curve $a$ is the same as $e$, with a factor 5 increase in vertical scale. The error bars to the left and right of $a$ show the expected fluctuations due to photoelectron counting statistics. It may be seen that the observed fluctuations are very closely the same. The increased noise at the continuum break, just longward of $H$ and $K$, is likely clue to increased counting noise in the very bright $[\mathrm{O} \mathrm{I}]$ nightsky line at $\lambda 5577$. The heavy curve $b$ is Spinrad's spectrum of the center of M31 redshifted and degraded in spectral resolution to match our galaxy spectrum. The dip near $\lambda .5000$ is due to a fleck of dirt on the face of the tube.
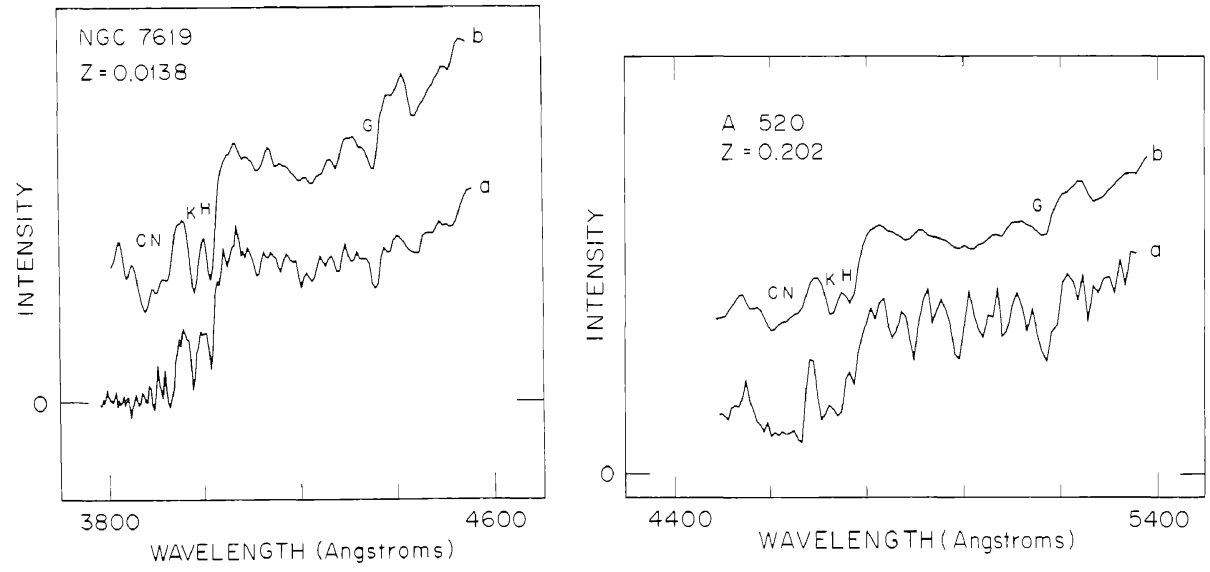

FIG. 2.-Sky subtracted spectra for galaxy clusters A520 and Peg I (NGC 7619). In each case, $a$ is the galaxy spectrum, after subtraction of the sky, and $b$ is Spinrad's spectrum for the center of M31, redshifted and degraded as in fig. 1. 
WESTPHAL, KRISTIAN, AND SANDAGE

TABLE 1

New Redshifts of Faint Galaxy Ciusters

\begin{tabular}{|c|c|c|c|c|}
\hline \multirow{2}{*}{ Ciuster } & \multicolumn{2}{|c|}{ Position* } & \multirow{2}{*}{$\begin{array}{c}\text { REDSHIFT, } \dagger \\
z=\Delta \lambda / \lambda_{0}\end{array}$} & \multirow{2}{*}{$\begin{array}{c}\text { OTHER REDSHIFTS, } \\
\text { Notes, AND } \\
\text { REFERENCES }\end{array}$} \\
\hline & $\alpha(1950)$ & $\delta(1950)$ & & \\
\hline $0024+1654 \ldots \ldots \ldots \ldots$ & $00^{\mathrm{h}} 24^{\mathrm{m}} 00^{\mathrm{s}}$ & $+16^{\circ} 53^{\prime} 24^{\prime \prime}$ & 0.392 & $0.38 \pm 0.01(\mathrm{~A})$ \\
\hline 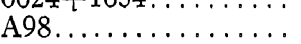 & 004351 & +201153 & 0.1042 & $0.1034(\mathrm{~B})$ \\
\hline $\mathrm{A} 222 \ldots \ldots \ldots \ldots \ldots$ & 013506 & -131516 & 0.216 & \\
\hline$\ldots \ldots \ldots \ldots$ & 013534 & -130034 & 0.205 & \\
\hline A $278 \ldots \ldots \ldots \ldots \ldots$ & 015422 & +320015 & 0.0891 & 0.0904 (C) \\
\hline $0221-0849 \ldots \ldots \ldots$ & 022131 & -084908 & 0.273 & \\
\hline$A 376 \ldots \ldots \ldots \ldots \ldots$ & $0242 \quad 59$ & +364150 & 0.0490 & $0.0487(\mathrm{C})$ \\
\hline$\ldots \ldots \ldots \ldots \ldots$ & 045127 & +024840 & 0.203 & \\
\hline$\ldots \ldots \ldots \ldots$ & 074602 & $\begin{array}{r}725436 \\
+72\end{array}$ & 0.226 & \\
\hline A795 $\ldots \ldots \ldots \ldots \ldots$ & 092121 & +142328 & 0.1357 & \\
\hline $0939+0912 \ldots \ldots \ldots$ & 093921 & +091200 & 0.206 & \\
\hline$A 963 \ldots \ldots \ldots \ldots \ldots$ & 101406 & $\begin{array}{r}+391739 \\
+\end{array}$ & 0.206 & \\
\hline$A 1146 \ldots \ldots \ldots \ldots \ldots$ & $10 \quad 5847$ & -222734 & 0.137 & $0.142 \pm 0.007(\mathrm{D})$ \\
\hline Peg I (NGC 7619) .... & $23 \quad 17 \quad 42$ & +075553 & 0.0133 & $0.0132(\mathrm{E})$ \\
\hline
\end{tabular}

* Positions measured by us for the galaxy whose redshift is given: this is the brightest cluster galaxy in all cases. Positions should be accurate to better than $15^{\prime \prime}$ in each coordinate.

$\dagger$ Redshifts corrected for galactic rotation: $\Delta z=\sin l^{\mathrm{II}} \cos b^{\mathrm{II}} / 1000$. When three decimal places are given, the accuracy of $z$ is better than 0.001 : when four decimal places are given, the accuracy is better than 0.0005 .

Notes And References.--(A) Baum 1962, Oke 1971; (B) Sargent 1973; (C) Peterson 1970; (D) unpublished continuum redshift by Kristian, using the 200 -inch multichannel scanner;

(E) Humason et al. 1956.

compare favorably with our experience using the regular 200 -inch Cassegrain image-tube spectrograph. We would expect that the quantum efficiencies of the photocathodes are comparable, but that the prism spectrograph has a throughput advantage of some tens of percent over a grating instrument. The data on exposure times are consistent with this expectation.

The major advantage of the SIT system over the image-tube spectrograph, however, is not speed, but storage capacity, reproducibility of the transfer function, and therefore the sky subtractive capability which permits fainter objects to be measured. The measurements at $z=0.4$ have given no more problems than those at $z=0$, except for the longer exposure times, and we anticipate no difficulty in going appreciably fainter.
In summary, it appears from these tests that a commercial SIT tube can be used in a straightforward way to obtain two-dimensional spectra with digitized, linear output. The data are nearly photon-noise limited. The sky can be obtained on the same exposure as the object, and can be subtracted to the photon noise level. Redshifts for faint galaxies were measured, and agree with conventional photographic values.

We plan to use the digital prism spectrograph described here to measure line redshifts for the faintest possible cluster galaxies in an effort to extend the Hubble diagram.

This work was supported in part by the National Science Foundation, under grant GP-36450X to the Carnegie Institution of Washington.

\section{REFERENCES}

Baum, W. A. 1962, IAU Symposium No. 15, ed. G. C. McVittie (New York: Macmillan), p. 390.

Beaver, E., and McIlwain, C. E. 1971, Rev. Sci. Instr., 42, 1321.

Glaspey, J. W., and Walker, G. A. H. 1973, Institute of Astronomy and Space Science, Vancouver.

Hubble, E. 1929, Proc. Nat. Acad. Sci., 15, 168.

Humason, M. L. 1929, Proc. Nat. Acad. Sci., 15, 167. 56,62 .

Humason, M. L., Mayall, N. U., and Sandage, A. R. 1956, A.J., 61,97 .
Kristian, J., and Westphal, J. A. 1975, in preparation.

Lowrance, J. L., and Zucchino, P. M. 1969, Advances in Electronics and Electron Physics, Vol. 28B, ed. L. Marton (New York: Academic Press), p. 851.

Oke, J. B. 1971, Ap. J., 170, 193.

Peterson, B. A. 1970, A.J., 75, 695.

Robinson, L., and Wampler, E. J. 1972, Pub. A.S.P., 84, 161.

Sargent, W. L. W. 1973, Pub. A.S.P., 85, 281.

Zucchino, P., and Lowrance, J. L. 1971, Astronomical Use of Television-Type Image Sensors, ed. V. R. Boscariuo (NASA SP-256), p. 27.

Jerome Kristian and Allan Sandage: Hale Observatories, 813 Santa Barbara Street, Pasadena, CA 91101 J. A. Westphal: California Institute of Technology, 1201 East California Blvd., Pasadena CA 91109 